\title{
A high serum phosphate and calcium-phosphate product is associated with cerebral small vascular disease in patients with stroke; a real world study.
}

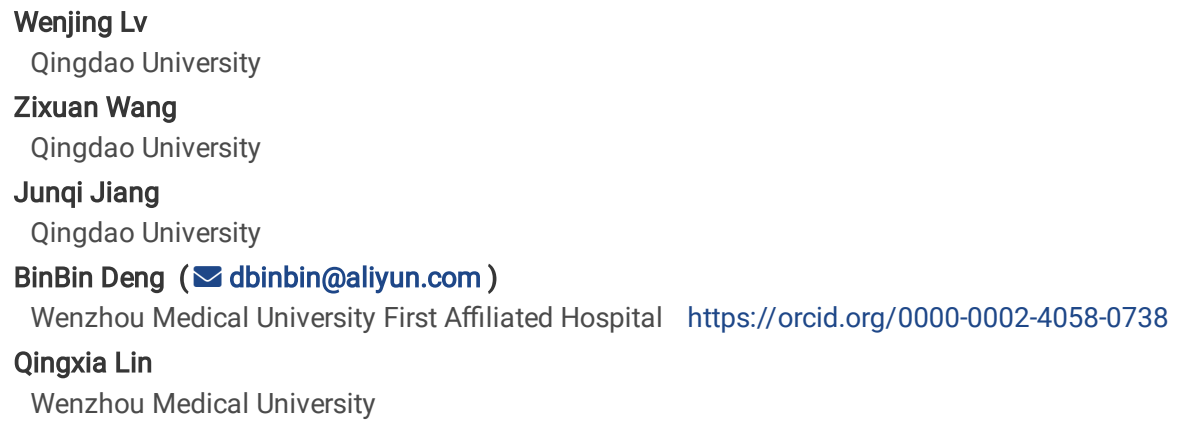

Version of Record: A version of this preprint was published at Frontiers in Nutrition on April 4th, 2022. See the published version at https://doi.org/10.3389/fnut.2022.801667. 


\section{Abstract}

Cerebral small vessel disease (CSVD) is a slowly progress disease, often accompanied by stroke, and result in dementia, depression, cognitive impairment, etc. It had already known that calcium and phosphorus metabolism (CPM) disorders were associated with vascular related adverse events. Serum Ca, $\mathrm{P}$, CaxP are the most commonly used indicators of CPM in clinical. The risk factors of CSVD and the relationship of serum Ca, P, CaxP and CSVD in stroke patients who do not have CPM disorders are still obscure. We enrolled 488 CSVD patients in a cohort from a consecutive hospital-based stroke registry, and further subgrouped them into lacunes, white matter hyperintensities (WMHs), cerebral microbleeds (CMBs), and matched them to 140 stroke patients without CSVD as controls. CSVD patients had higher levels of serum Ca, P, CaxP. We developed 2 predictive models and nomograms (incorporating age, hypertension, $\mathrm{P}$ or CaxP, respectively) for CSVD patients. The prediction and calibration power were good. Area under the curve (AUC) was $0.855,0.851$, respectively. $P$ value of Hosmer-Lemeshow goodness of fit (H-L) test was $0.689,0.698$, respectively. P and CaxP were positive correlated with CSVD, with OR 3720.401 (646.66521404.249), 1.294 (1.222-1.370), respectively. We further validated the models in lacunes, WMHs, and CMBs, and found models were still valid in these 3 subtypes. In summary, a high serum P or CaxP are associated with an increased risk of CSVD in stroke patients who without CPM disorders.

\section{Introduction}

Cerebral small vessel disease (CSVD) is a very common neurological whole-brain disease in older people, which results from pathologies in cerebral arterioles, capillaries and venules. It causes up to $45 \%$ of dementia, $20 \%$ of all stroke, and cognitive impairment, depression and gait problems are also frequently seen in CSVD patients (Pantoni, 2010). The diagnosis of CSVD has relied on imaging findings including lacunes, white matter hyperintensities (WMHs), and microbleeds (CMBs) (Shi \& Wardlaw, 2016). Epidemiology of CSVD might differ in Asian compared with non-Asia population (Shi \& Wardlaw, 2016). CSVD has a robust association with large artery atherosclerosis, and often accompanies by stroke in USA (Del Brutto et al., 2020). It is often ignored when accompanied by stroke among inpatients, since its manifestation is less devastating and progress slower than large vessel occlusion. Few studies investigate the epidemiology of CVSD accompanied by stroke in China. Antiplatelet and management of traditional risk factors still remain the most important therapeutic and preventive approach, due to limited understanding of CSVD, however, vascular risk factors and large artery disease explain quite small part of the variance of CSVD (Wardlaw et al., 2014). Exploring the risk factor of CSVD is necessary for targeted prevention and treatment of CSVD.

Artery calcification is part of the atherosclerotic process, it is considered as ectopic deposition of bone components and is highly associated with WMHs, lacunes, and CMBs (Chen et al., 2019). Serum phosphate (P), calcium (Ca) and calcium-phosphate product (CaxP) is calcium and phosphorus metabolism (CPM)-related factors. In extreme cases, such as chronic kidney diseases (CKD), CPM homeostasis disruption can promote ectopic vascular calcification in blood vessel, including elevated P levels and increased CaxP (Reiss et al., 2018). Lowering dialysate Ca concentration can improve the aortic stiffness and decrease the carotid intima-media thickness (He et al., 2016). However, CPM disorders are not quite common risk factors in stroke, and less attention has been paid to the potential involvement of CPM in CSVD patients who without CPM disorders.

\section{Materials And Methods}

This study was approved by the Ethics Committee of the First Affiliated Hospital of Wenzhou Medical University. The experiments were undertaken with the understanding and written consent of each subject, and that the study conforms with World Medical Association Declaration of Helsinki. From 2016.4 .1 to 2018.7.31, there were 1102 ischemic stroke patients were admitted to the First Affiliated Hospital of Wenzhou Medical University. 588 of them were included in this study. 140 of whom had no CSVD signs served as 'no CSVD' group, 448 of whom had any signs of CSVD in the cranial MR images served as 'CSVD' group. 448 of the include cases had lacunes, 212 of them had scattered lacunes, and 236 of them had multiple lacunes. 100 of the included cases had WMHs, and 55 of the included cases had CMBs. Cases with overlapping subtypes were repeated included in each subtype.

\section{Exclusion criteria}

Patients lacked of serum Ca and P tests. Patients had any diseases that could influence CPM, including chronic kidney disease with blood creatinine level>186 $\mu \mathrm{mol} / \mathrm{L}$, bone metastasis of malignant tumor, parathyroid disease, pituitary disorder. The demographic, chronic disease, hematologic parameters, and imaging findings of all patients were collected using standardized data records.

\section{Clinical and laboratory assessments}

All blood indicators were assessed on the morning of the second day of admission after overnight fasting. Cranial MR examinations were completed within 3 days of admission. Vascular risk factors of cases were collected according to patient description.

\section{Classification criteria}

Lacunes are shown as hypointense lesions with hyperintense rim on FLARE and T1, with hyperintense signal on T2. The diameters are 3-15mm. It can be rounded, ovoid or tubular, and can distributed in the white matter, basal ganglia, pons or brainstem. Recent small ischemic lacunes is included, that are best identified on DWI, as hyperintense on DWI囚T2 and FLAIR, hypointense on T1, with usual diameter $\leq 20 \mathrm{~mm}$. WMHs are usually symmetrically and bilaterally distributed in the white matter including the pons and brain stem, and also occur in deep grey matter. They appear hyperintense to the normal brain on T2 or FLAIR, and can be patchy or confluent. CMBs are regarded as small round and homogeneous foci of hypointensity on T2-weighted MRI and susceptibilityweighted imaging. CSVDs are with single or combined signs of lacunes, WMHs and CMBs (Shi \& Wardlaw, 2016).

\section{Statistical analysis}


All baseline data and risk factors of excluded and include cases were statistically analyzed by logistic regression, using the probability as propensity score. Their distribution were viewed in a population pyramid graph. For baseline data and risk factors of included cases, categorical variables were compared with the chi-square test, and continuous variable were compared with the Mann-Whitney $U$ test, $p$-value $<0.05$ was considered statistically significant. Logistics regression analysis of univariate was used for identifying risk factors, and factors with $p$ value $<0.1$ were analyzed in multivariate analysis with logistic regression, with a method of Backward LR. The nomograms were constructed performed with R. After logistic regression analysis and calculation of probabilities in multivariate analysis, we assessed the performance of the model by calculating the AUC. The larger the AUC, the more accurate the prognosis is. $\mathrm{H}-\mathrm{L}$ test was used to evaluate the calibration degree of models, models with $\mathrm{p}$ value $>0.1$ were considered in good calibration degree. All calculations were based on SPSS version 22.0 software and $\mathrm{R}$.

\section{Results}

Clinical information of 1102 stroke patients with cranial MR were collected, including demographic parameters (age, gender, weight, ), vascular risk factors (hypertension, diabetes, coronary artery disease, atrial fibrillation, hyperlipidemia, history of smoking and drinking alcohol), blood biochemistry and vital signs on admission (NIHSS score, MBP, FGB, P2hPG, HbA1c, TC, TG, LDL, HDL, LVEF, WBC, Neutrophil, Lymphocyte, RBC,HB, TP, Albumin, PLT, PT, INR, APTT, FIB,BUN, Cr, HCY, TSH, FT3, FT4), serum biochemical markers of CPM (P, Ca, adjusted Ca, CaxP, adjusted CaxP). Patients lack of serum Ca and P data, or with CPM disorders, such as severe chronic kidney disease, pituitary or parathyroid disease, bone metastasis of malignant tumor, were excluded (Fig. 1). In order to check if there was selection bias, we calculated propensity scores of demographic parameters, vascular risk factors, blood biochemistry and vital signs on admission in excluded and included cases respectively. Distributions of propensity score were similar between groups (Fig. 2).

Patients with any one or more image representations of lacunes, WMHs, CMBs were divided into CSVD group, and patients without CSVD signs were divided into no CSVD group. There were 140 cases in no CSVD group and 448 cases in CSVD group. Up to $76.19 \%$ of stroke patients, had signs of CSVD on cranial MR images (Fig. 3A). TOAST classification distributions had no statistical difference among no CSVD and CSVD groups (Fig. 4). The general characteristics of two groups were detailed in Table 1. Age, rate of hypertension or diabetes, serum P, Ca, CaxP, adjusted CaxP were higher, and WBC, HB were lower in CSVD patients than no CSVD patients (Table 1, Fig. 5).

Univariate and multivariate logistic regression were used to identify factors potentially prognostic factors of CSVD, and further construct models to predict CSVD. Receiver operating characteristic (ROC) curves were used as the discrimination index of models. Hosmer-Lemeshow goodness of fit (H-L) test were used to evaluate the calibration degree of models. All factors with $p$ value $<0.2$ in table 1 were analyzed by univariate logistic regression (Table 3 ). Baseline factors with $p$ value $<0.1$ in table 3 , including age, hypertension, diabetes, MBP, WBC, RBC, HB, BUN were analyzed with multivariate logistic regression, and factors with $p$ value $<0.05$, including age, hypertension, diabetes, MBP, WBC, BUN were included in model 1 ultimately (Table 2), AUCs is 0.771 and $p$ value of $\mathrm{H}-\mathrm{L}$ test is $0.873>0.05$ (Fig.3).

Based on model 1, we constructed model 2 (including P, Ca or adjusted Ca), model 3 (including CaxP), model 4 (including adjusted CaxP) (Table 2). In these three models, only age and hypertension were still remained in the end. $\mathrm{P}$ was included in model 2, CaxP was included in model 3 , and adjusted CaxP was included in model 4 (Table 2). AUCs were similar among three models, $0.855,0.851,0.853$, respectively, better than model 1 (Fig. $6 \mathrm{~A}$ ). $\mathrm{P}$ value of $\mathrm{H}$ - $\mathrm{L}$ test is $0.689,0.698,0.307$, respectively (Fig. 6B). Adjusted CaxP impaired the calibration degree in model 4 , and was no better than CaxP. hence, there was no need to use adjusted CaxP to predict CSVD instead of CaxP. Model 2 and model 3 had similar discrimination and calibration power, thus, we selected them to establish nomograms to predict the probability of CSVD (Fig. 7). Age, hypertension, P or CaxP were all positive factors related to CSVD. The OR of P was 3720.401 (646.665-21404.249), and OR of CaxP was (1.294, 1.222-1.370) (Table. 2). P, CaxP are potential CSVD risk factors.

We explored the incidence of subtypes of CSVD, including lacunes, WMHs, and CMBs, and patients with lacunes were further divided into scattered lacunes and multiple lacunes. Then we validate if model 2 and model 3 were valid in these subtypes. We found that lacunes was the most common types in CSVD, $22.96 \%$ of the CSVD patients have two or three types coexisted (Fig. 4A). P and CaxP were significantly increased in lacunes than no CSVD, and was highest in multiple lacunes. Ca is slightly higher in multiple lacunes than no CSVD. P and CaxP were significantly increased in WMHs and CMBs, compared with no CSVD. Ca were slightly increased in WMHs than no CSVD (Fig. 4B-2D). P and CaxP were still positive risk factors in each subgroup of CSVD (Table 4-6). AUCs and $\mathrm{H}$-L test shows good discrimination and calibration power of models in each subgroup (Fig. 3, Table. 7).

\section{Discussion}

Using a hospital stroke registry of the First Affiliated Hospital of Wenzhou Medical, we found that $76.19 \%$ of relatively normal kidney function stroke patients concomitant with CSVD. Higher serum P and CaxP levels were positively linked to CSVD, and subtypes of CSVD, including lacunes, WMHs, and CMBs. Most CPM related clinical investigations are conducted in chronic kidney disease population, and takes large blood vessel calcification as research target(Felsenfeld, Levine, \& Rodriguez, 2015; Reiss et al., 2018). High P and Ca could promote large vessel calcification, and their influences can mutually reenforcing (Fu, Cui, Ning, Fu, \& Liao, 2015; Masumoto et al., 2017; Panizo et al., 2016). CSVD is concomitant with stroke in South China, indicating they may share some common risk factors or pathologies, although they are two different kinds of diseases. Our results indicates that higher serum $\mathrm{P}$ and CaxP may promote CSVD even when they are in normal levels. Studies of CSVD and CPM has some discrepancies in different regions. A study in Taiwan shows that circulatory P levels $>3.925 \mathrm{mg} / \mathrm{dL}$ were associated with severe WMHs in a community-based longitudinal aging Study(Chung et al., 2019). A study in Turkey follows subjects who underwent brain imaging for any reason, reported that CSVD patients had lower P than normal subjects (Ozelsancak, Micozkadioglu, Torun, \& Tekkarismaz, 2019). In US veterans population, high serum P is associated with CSVD and dementia, and this relationship is more significantly in participants $<60$ years old ( $\mathrm{Li}, \mathrm{Xie}$, Bowe, Xian, \& Al-Aly, 2017). CPM is significant affected by light and diet in normal population, further large-scale cohort studies on relationship of CPM and CSVD tracking of localized community are need. 
The primary underlying initiating cause of CSVD is the derangement of the blood-brain barrier (BBB), this may start some years before the first symptoms, leads to the small vessel structural changes and perivascular changes (Liu, Yang, \& Fan, 2020), the cellular structure of the BBB is mainly composed of brain microvascular endothelial cells (MVECs) (Wardlaw, 2010). Junction protein (zona occludens-1, occludin, and claudin-5) expression is downregulated in human brain MVECs after high phosphorus treatment (Chung et al., 2019). Many BBB dysfunction diseases are associated with increasing intracellular Ca levels of MVECs and serum Ca. Many cell membrane ion channels are responsible for regulating intracellular Ca balance. Plasma membrane calcium ATPase could export calcium, and calcium entry from the extracellular space can occur from store-operated cation channels or receptor-operated cation channels (Dalal, Muller, \& Sullivan, 2020). In addition, voltage-gated L-type calcium channels and the transient receptor potential superfamily have been identified to comprise calcium influx channels in endothelial cell (Jiang et al., 2018).

High levels of phosphate accelerate the precipitation of calcium and phosphate in the form of hydroxyapatite, accelerating vascular calcification (Reynolds et al., 2004). Calcification is associated independently with high calcium levels, and is synergistic with elevated phosphate levels (Shroff et al., 2010) (Schurgers et al., 2018). High phosphate can directly induces phenotypic transformation of vessel smooth muscular cells (VSMCs) into osteoblasts by stimulating VSMCs to express core binding factor-a1, and repress the production of calcification inhibitors and promote the release of extracellular vesicles (EVs) lacking these inhibitors, but rich in pro-calcific proteins such as tissue-nonspecific alkaline phosphatase (Yang et al., 2019). miR-29b, miR-133b, and miR-211 have direct roles in the vascular smooth muscle calcification induced by high phosphorus (Panizo et al., 2016). In VSMCs, the sodium-phosphorus co-transporter PiT-1 promotes matrix calcification caused by elevated phosphorus, while PiT-2 inhibits its changes (Yamada \& Giachelli, 2017). Vitamin K-dependent matrix Gla protein (MGP) is a key inhibitor in the formation of vascular calcification, and elevated phosphate and calcium levels increased MGP levels, as an inherent protective mechanism of vascular calcification (Houben, Neradova, Schurgers, \& Vervloet, 2016).

Although this study showed propensity score distribution of the included and excluded data to confirm that the baseline characteristic of the excluded cases was similar to that of the included cases. However, the analyzed data was from a single-center medical institution in Southern China. The population in the hospital had higher health literacy, compared to those who did not seek medical attention; the selected population had geographic and ethnic specificities; awareness rate of vascular risk factors varied. All above factors lead to an inevitable selection bias and recalling bias of the study. Further multi-center, multiethnic, community-based research should be conducted.

In conclusion, serum $\mathrm{P}$ and CaxP was higher in CSVD patients, including subtypes of lacunes, especially multiple lacunes, WMHs, and CMBs. Patients with elder age, hypertension, higher $\mathrm{P}$ or CaxP had an increased risk of CSVD in stroke patients who did not have CPM disorder. Higher $\mathrm{P}$ or CaxP are also risk factors of CSVD subtypes, including lacunes, WMHs, and CMBs.

\section{Abbreviations}

Mean blood pressure (MBP); left ventricular ejection fraction (LVEF); fasting blood glucose (FBG); 2-hour post-meal blood glucose (P2hPG); glycated hemoglobin ( $\mathrm{HbA} 1 \mathrm{c} \%)$; total cholesterol (TC); triglyceride (TG); low density lipoprotein cholesterol (LDL); high density lipoprotein cholesterol (HDL); white blood cell (WBC); red blood cell (RBC); blood platelet (PLT); hemoglobin (HB); total serum protein (TP); total prothrombin time (PT); international normalized ratio (INR); activated partial thromboplastin time (APTT); fibrinogen (FIB); urea nitrogen (BUN); creatinine (Cr); homocysteine (HCY); thyroid stimulating hormone (TSH); free triiodothyronine (FT3); free tetraiodothyronine (FT4); National Institutes of Health Neurological Deficit Score (NIHSS); cerebral small vascular disease (CSVD); white matter hyperintensities (WMHs).; cerebral microbleeds (CMBs), calcium and phosphorus metabolism (CPM); chronic kidney diseases (CKD); microvascular endothelial cells (MVECs); vessel smooth muscular cells (VSMCs); extracellular vesicles (EVs); matrix Gla protein (MGP)

\section{Discussion}

Funding: This work was supported by the National Natural Science Foundation of China (81901273) and Science Technology Department of Zhejiang Province (Grant no. Q21H090076).

Conflicts of interest/Competing interests: The authors have stated explicitly that there are no conflicts of interest in connection with this article.

Ethics approval: The Ethical Decision Committee of the Research Administration at First Affiliated Hospital of Wenzhou Medical University approved the study (CR2009041).

Consent to participate: All patients agreed to participate in the study, and use their clinic data and information for research purpose.

Consent for publication: All participants agreed for publications related to this study.

Availability of data and material: Data and material can be shared with the consent of corresponding authors.

Code availability: SPSS version 22.0 software and R.

Authors' contributions: Wenjing Lv and Zixuan Wang were responsible for data statistics and writing paper. Junqi Jiang collected data. Binbin Deng and Qingxia Lin provided resources and designed the study.

\section{References}

Chen, Y. C., Wei, X. E., Lu, J., Qiao, R. H., Shen, X. F., \& Li, Y. H. (2019). Correlation Between Intracranial Arterial Calcification and Imaging of Cerebral Small Vessel Disease. Front Neurol, 10, 426. doi: 10.3389/fneur.2019.00426

Page $4 / 12$ 
Chung, C. P., Peng, L. N., Chou, K. H., Liu, L. K., Lee, W. J., Lin, C. P., .. Wang, P. N. (2019). High Circulatory Phosphate Level Is Associated with Cerebral SmallVessel Diseases. Trans/ Stroke Res, 10(3), 265-272. doi: 10.1007/s12975-018-0639-6

Dalal, P. J., Muller, W. A., \& Sullivan, D. P. (2020). Endothelial Cell Calcium Signaling during Barrier Function and Inflammation. Am J Pathol, $190(3)$, 535-542. doi: 10.1016/j.ajpath.2019.11.004

Del Brutto, O. H., Mera, R. M., Del Brutto, V. J., Hill, J. P., Torpey, A. P., Peralta, L. D., . . Sedler, M. J. (2020). Cerebral small vessel disease score and atherosclerosis burden - A population study in community-dwelling older adults. Clin Neurol Neurosurg, 194, 105795. doi: 10.1016/j.clineuro.2020.105795

Felsenfeld, A. J., Levine, B. S., \& Rodriguez, M. (2015). Pathophysiology of Calcium, Phosphorus, and Magnesium Dysregulation in Chronic Kidney Disease. Semin Dial, 28(6), 564-577. doi: 10.1111/sdi.12411

Fu, X., Cui, Q. Q., Ning, J. P., Fu, S. S., \& Liao, X. H. (2015). High-Flux Hemodialysis Benefits Hemodialysis Patients by Reducing Serum FGF-23 Levels and Reducing Vascular Calcification. Med Sci Monit, 21, 3467-3473. doi: 10.12659/msm.894894

He, Z., Cui, L., Ma, C., Yan, H., Ma, T., \& Hao, L. (2016). Effects of Lowering Dialysate Calcium Concentration on Carotid Intima-Media Thickness and Aortic Stiffness in Patients Undergoing Maintenance Hemodialysis: A Prospective Study. Blood Purif, 42(4), 337-346. doi: 10.1159/000450747

Houben, E., Neradova, A., Schurgers, L. J., \& Vervloet, M. (2016). The influence of phosphate, calcium and magnesium on matrix Gla-protein and vascular calcification: a systematic review. G Ital Nefrol, 33(6).

Jiang, X., Andjelkovic, A. V., Zhu, L., Yang, T., Bennett, M. V. L., Chen, J., .. Shi, Y. (2018). Blood-brain barrier dysfunction and recovery after ischemic stroke. Prog Neurobiol, 163-164, 144-171. doi: 10.1016/j.pneurobio.2017.10.001

Li, T., Xie, Y., Bowe, B., Xian, H., \& Al-Aly, Z. (2017). Serum phosphorus levels and risk of incident dementia. PLoS One, 12(2), e0171377. doi: $10.1371 /$ journal.pone.0171377

Liu, Q., Yang, Y., \& Fan, X. (2020). Microvascular pericytes in brain-associated vascular disease. Biomed Pharmacother, 121, 109633. doi: 10.1016/j.biopha.2019.109633

Masumoto, A., Sonou, T., Ohya, M., Yashiro, M., Nakashima, Y., Okuda, K., . . Shigematsu, T. (2017). Calcium Overload Accelerates Phosphate-Induced Vascular Calcification Via Pit-1, but not the Calcium-Sensing Receptor. J Atheroscler Thromb, 24(7), 716-724. doi: 10.5551/jat.36574

Ozelsancak, R., Micozkadioglu, H., Torun, D., \& Tekkarismaz, N. (2019). Cerebrovascular events in hemodialysis patients; a retrospective observational study. BMC Nephrol, 20(1), 466. doi: 10.1186/s12882-019-1629-y

Panizo, S., Naves-Diaz, M., Carrillo-Lopez, N., Martinez-Arias, L., Fernandez-Martin, J. L., Ruiz-Torres, M. P., . . Rodriguez, I. (2016). MicroRNAs 29b, 133b, and 211 Regulate Vascular Smooth Muscle Calcification Mediated by High Phosphorus. J Am Soc Nephrol, 27(3), 824-834. doi: 10.1681/ASN.2014050520

Pantoni, L. (2010). Cerebral small vessel disease: from pathogenesis and clinical characteristics to therapeutic challenges. Lancet Neurol, 9(7), 689-701. doi: $10.1016 /$ S1474-4422(10)70104-6

Reiss, A. B., Miyawaki, N., Moon, J., Kasselman, L. J., Voloshyna, I., D'Avino, R., Jr., \& De Leon, J. (2018). CKD, arterial calcification, atherosclerosis and bone health: Inter-relationships and controversies. Atherosclerosis, 278, 49-59. doi: 10.1016/j.atherosclerosis.2018.08.046

Reynolds, J. L., Joannides, A. J., Skepper, J. N., McNair, R., Schurgers, L. J., Proudfoot, D., . . Shanahan, C. M. (2004). Human vascular smooth muscle cells undergo vesicle-mediated calcification in response to changes in extracellular calcium and phosphate concentrations: a potential mechanism for accelerated vascular calcification in ESRD. J Am Soc Nephrol, 15(11), 2857-2867. doi: 10.1097/01.ASN.0000141960.01035.28

Schurgers, L. J., Akbulut, A. C., Kaczor, D. M., Halder, M., Koenen, R. R., \& Kramann, R. (2018). Initiation and Propagation of Vascular Calcification Is Regulated by a Concert of Platelet- and Smooth Muscle Cell-Derived Extracellular Vesicles. Front Cardiovasc Med, 5, 36. doi: 10.3389/fcvm.2018.00036

Shi, Y., \& Wardlaw, J. M. (2016). Update on cerebral small vessel disease: a dynamic whole-brain disease. Stroke Vasc Neurol, 1(3), 83-92. doi: 10.1136/svn2016-000035

Shroff, R. C., McNair, R., Skepper, J. N., Figg, N., Schurgers, L. J., Deanfield, J., . . Shanahan, C. M. (2010). Chronic mineral dysregulation promotes vascular smooth muscle cell adaptation and extracellular matrix calcification. J Am Soc Nephrol, 21(1), 103-112. doi: 10.1681/ASN.2009060640

Wardlaw, J. M. (2010). Blood-brain barrier and cerebral small vessel disease. J Neurol Sci, 299(1-2), 66-71. doi: 10.1016/j.jns.2010.08.042

Wardlaw, J. M., Allerhand, M., Doubal, F. N., Valdes Hernandez, M., Morris, Z., Gow, A. J., . . Deary, I. J. (2014). Vascular risk factors, large-artery atheroma, and brain white matter hyperintensities. Neurology, 82(15), 1331-1338. doi: 10.1212/WNL.0000000000000312

Yamada, S., \& Giachelli, C. M. (2017). Vascular calcification in CKD-MBD: Roles for phosphate, FGF23, and Klotho. Bone, 100, 87-93. doi:

10.1016/j.bone.2016.11.012 
Yang, W., Zou, B., Hou, Y., Yan, W., Chen, T., \& Qu, S. (2019). Extracellular vesicles in vascular calcification. Clin Chim Acta, 499, 118-122. doi: 10.1016/j.cca.2019.09.002

\section{Tables}

Table 1 The general characteristics of no CSVD and CSVD patients.

\begin{tabular}{|c|c|c|c|}
\hline Baseline characteristics & No CSVD & CSVD & P-value \\
\hline N & 140 & 448 & \\
\hline $\begin{array}{l}\text { Age, years } \\
\text { Femal }\end{array}$ & $\begin{array}{l}58.82 \pm 12.99 \\
45(32.14 \%)\end{array}$ & $\begin{array}{l}70(62,77) \\
155(34.59 \%)\end{array}$ & $\begin{array}{l}.000 \\
.661\end{array}$ \\
\hline Hypertension & $100(71.43 \%)$ & $393(88.51 \%)$ & .000 \\
\hline Diabetes & $39(2$ & $181(40.58 \%)$ & .007 \\
\hline Coronary artery desease & $18(13.95 \%)$ & $76(18 \%)$ & .349 \\
\hline $\begin{array}{l}\text { Atrial fibrillation } \\
\text { Hyperlipidemia }\end{array}$ & $\begin{array}{l}19 \square 13.57 \% \square \\
69 \square 49.64 \% \square\end{array}$ & $\begin{array}{l}68 \square 15.21 \% \square \\
216 \square 48.98 \% \square\end{array}$ & $\begin{array}{l}.685 \\
.923\end{array}$ \\
\hline History of smoking & $69 \square 49.29 \% \square$ & $191 \square 42.73 \% \square$ & .174 \\
\hline alcohol & 54 & $147 \square 32.89 \% \square$ & .222 \\
\hline Weight, kg & $65(60,69.8)$ & $65(59,70)$ & .369 \\
\hline MBP, mmHg & $104.04 \pm 14.63$ & $\begin{array}{l}2.5(1,4) \\
106.67(97,12.67)\end{array}$ & $\begin{array}{l}.864 \\
.082\end{array}$ \\
\hline & 5.1 & $5.2(4$ & .655 \\
\hline $\begin{array}{l}\text { P2hPG, mmol/L } \\
\text { HbA1c \% }\end{array}$ & (3,11.84) & $\begin{array}{l}37,11.3) \\
7)\end{array}$ & .281 \\
\hline $\mathrm{hol} / \mathrm{L}$ & .08) & $.79,5.32)$ & .612 \\
\hline & 1.6 & $1.5(1$. & .146 \\
\hline LDL, $\mathrm{mmol} / \mathrm{L}$ & $\begin{array}{l}2.55(2.07,3.07) \\
0.99(0.84,1.178)\end{array}$ & $\begin{array}{l}2.57(2.08,3.2) \\
1.04(0881.22)\end{array}$ & .521 \\
\hline LVEF, \% & $65<6$ & ,68.6) & \\
\hline WBC, $\times 10^{9} / \mathrm{L}$ & $7.01(5.66,8.55)$ & $6.54(5.43,7.82)$ & .023 \\
\hline Neutrophil, $\times 10^{9} / \mathrm{L}$ & $4.34(3.365,5.64)$ & $4.02(3.15,5.33)$ & .080 \\
\hline Lymphocyte, $\times 10^{9} / \mathrm{L}$ & $1.7(1.32,2.18)$ & $.69(1.31,2.05)$ & .529 \\
\hline $\mathrm{RBC}, \times 10^{12} / \mathrm{L}$ & $4.55(4.24,4.83)$ & $4.5(4.11,4.83)$ & .080 \\
\hline $\begin{array}{l}\mathrm{HB}, \mathrm{g} / \mathrm{L} \\
\mathrm{TP}, \mathrm{g} / \mathrm{L}\end{array}$ & $139(128.25,148.75)$ & $135(124,146)$ & .032 \\
\hline Albumin, $g / L$ & $\begin{array}{l}39.58) \\
309\end{array}$ & $9.9)$ & \\
\hline PLT, $\times 10^{9} / \mathrm{L}$ & 17250 & $(184,262)$ & .13 \\
\hline & $3.1,14.28)$ & 13.5( & .367 \\
\hline INR & $(0.99,1.11)$ & $1.03(0.98,1.09)$ & .483 \\
\hline $\begin{array}{l}\text { APTT, s } \\
\text { FIB }, / L\end{array}$ & $\begin{array}{l}36.9(34.03,39.58) \\
3.48(2.98,4.22)\end{array}$ & $\begin{array}{l}37.1(34.9,39.6) \\
3.63(296441)\end{array}$ & $\begin{array}{l}.358 \\
271\end{array}$ \\
\hline BUN, $\mathrm{mmol} / \mathrm{L}$ & $4.8(3.8,6)$ & $5(4.1,6.2)$ & .083 \\
\hline $\mathrm{Cr}, \mu \mathrm{mol} / \mathrm{L}$ & , 83.75) & 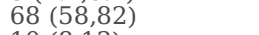 & \\
\hline $\mathrm{HCY}, \mu \mathrm{g} / \mathrm{L}$ & 10 & $10(8,13$ & .055 \\
\hline FT: & $\begin{array}{l}1.46(0.99,2 \\
4.4(4,4.78)\end{array}$ & $\begin{array}{l}1.62(1 \\
4.5(4.1\end{array}$ & \\
\hline FT4 & $10.21,12.79)$ & $(10.14,12.62$ & .588 \\
\hline$P, r$ & $73,0.98)$ & 10 & .00 \\
\hline $\mathrm{nmol} / \mathrm{L}$ & & 2.26 & .022 \\
\hline Adjusted $\mathrm{Ca}^{*}$ & 0.10 & $2.23(2.17,2.29)$ & .135 \\
\hline $\mathrm{Ca} \times \mathrm{P},(\mathrm{mg} / \mathrm{dL})^{2}$ & $21.36(19.1,26.2$ ? & $28.94(25.64,32.98)$ & .000 \\
\hline Adjusted CaxP, $(\mathrm{mg} / \mathrm{dL})^{2}$ & $21.84(20.09,26.29)$ & $29.22(25.98,33.28)$ & .000 \\
\hline
\end{tabular}

Normally distributed data is represented by mean \pm SD, skewed distribution data is represented by median (IQR), and frequency data is represented by $\mathrm{n}(\%)$. Adjusted Ca $(\mathrm{mmol} / \mathrm{L})=\mathrm{Ca}(\mathrm{mmol} / \mathrm{L})+0.02[40-$ Albumin $(\mathrm{g} / \mathrm{L})]$, serum Ca is adjusted if albumin $<35 \mathrm{~g} / \mathrm{L}$, or $>$ $51 \mathrm{~g} / \mathrm{L}$. Factors with $\mathrm{P}<0.05$ is statistically significant.

Table 2 Multivariate analysis for models by Binary Logistic regression.

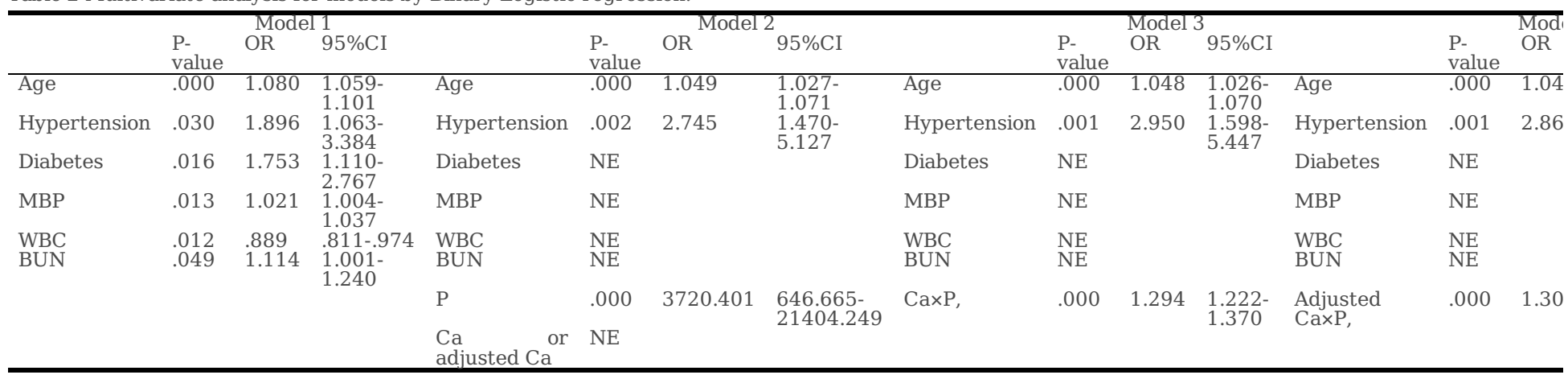

Model 1: adjusted for covariates that $\mathrm{p}<0.1$ in univariate analysis. Model $2=$ model $1+\mathrm{P}, \mathrm{Ca}$ or adjusted Ca. Model3 $=$ model $1+\mathrm{Ca} \times \mathrm{P}$. Model4 = model 1+ adjusted Ca $\times$ P. A method of Backward:LR are used, and factors with p value>0.05 are removed from models. NE: not entry.

Table 3. Univariate analysis for factors associated with CSVD by Binary Regression. 


\begin{tabular}{llll}
\hline Baseline characteristics & OR & $95 \% \mathrm{CI}$ & P-value \\
\hline N140/448 & 1.077 & $1.058-1.097$ & .000 \\
Age, years & 3.090 & $1.934-4.937$ & .000 \\
Hypertension & 1.769 & $1.168-2.679$ & .007 \\
Diabetes & .772 & $.527-1.129$ & .182 \\
History of smoking & 1.015 & $1.002-1.029$ & .027 \\
MBP, mmHg & 1.566 & $.796-3.080$ & .194 \\
HDL, mmol/L & .930 & $.857-1.010$ & .086 \\
WBC, $\times 10^{9} / \mathrm{L}$ & .941 & $.862-1.029$ & .182 \\
Neutrophil, $\times 10^{9} / \mathrm{L}$ & .714 & $.512-.995$ & .047 \\
RBC, $\times 10^{12} / \mathrm{L}$ & .988 & $.977-.999$ & .039 \\
HB, $\mathrm{g} / \mathrm{L}$ & 1.002 & $.999-1.005$ & .114 \\
PLT, $\times 10^{9} / \mathrm{L}$ & 1.090 & $.993-1.196$ & .069 \\
BUN, $\mathrm{mmol} / \mathrm{L}$ & 8473.940 & $1522.947-47150.476$ & .000 \\
P, mmol/L & 5.190 & $1.025-26.290$ & .047 \\
Ca, mmol/L & 3.118 & $.514-18.928$ & .217 \\
Adjusted Ca ${ }^{*}, \mathrm{mmol} / \mathrm{L}$ & 1.335 & $1.262-1.412$ & .000 \\
Ca×P, $(\mathrm{mg} / \mathrm{dL})^{2}$ & 1.345 & $1.269-1.425$ & .000 \\
Adjusted Ca×P, (mg/dL) ${ }^{2}$ & & & \\
\hline
\end{tabular}

Factors with $\mathrm{p}$ value $<0.2$ are listed.

Table 4. Multivariate analysis for factors associated with lacunes by Multinominal Regression.

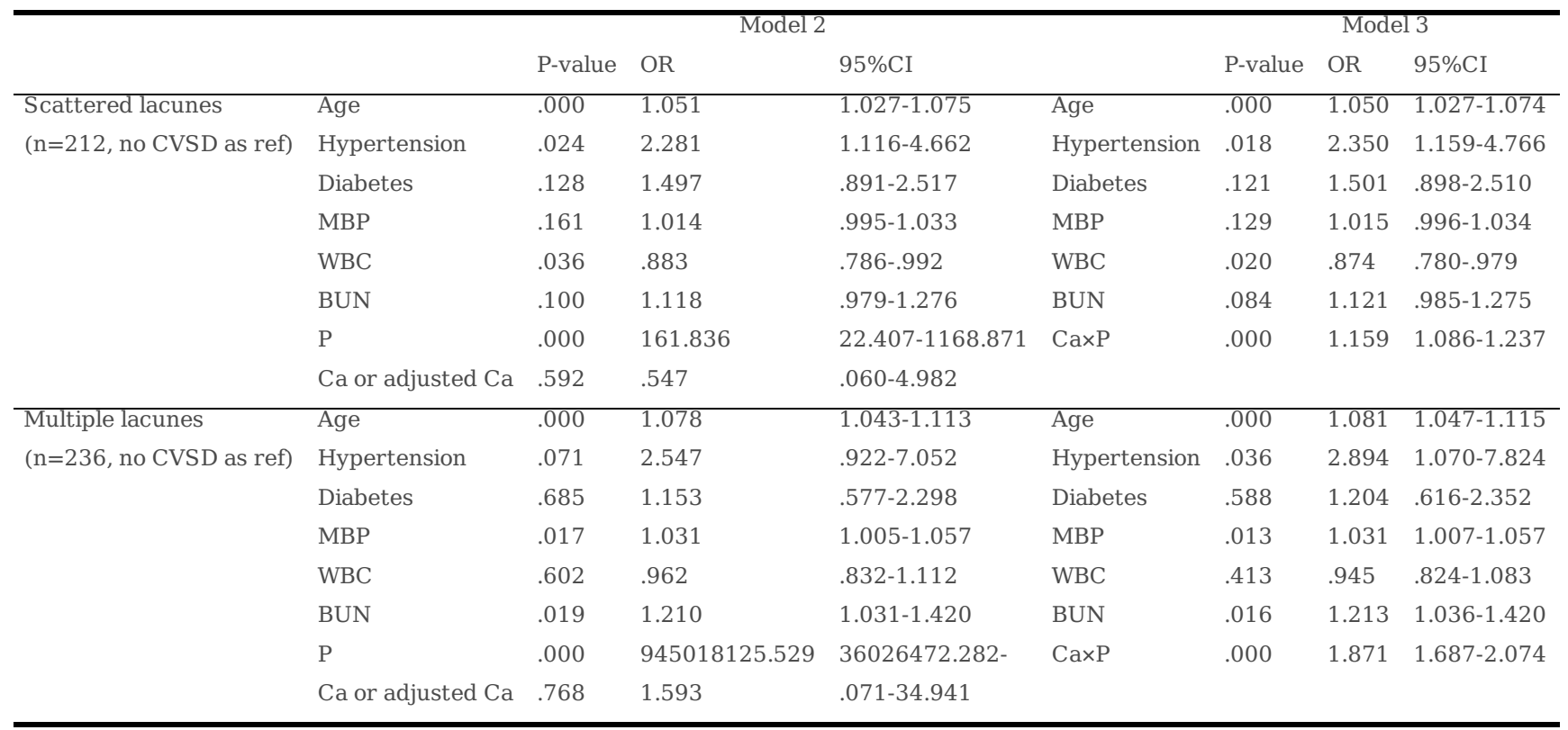

Factors with $\mathrm{P}$ value $<0.05$ entered into models.

Table 5. Multivariate analysis for factors associated with WMHs by Binary Regression.

\begin{tabular}{llllllll}
\hline & & \multicolumn{2}{c}{ Model 2 } & & \multicolumn{3}{c}{ Model3 } \\
& P-value & OR & 95\%CI & & P-value & OR & $95 \%$ CI \\
\hline Age & .000 & 1.122 & $1.071-1.176$ & Age & .000 & 1.118 & $1.068-1.171$ \\
Hypertension & $\mathrm{NE}$ & & & Hypertension & .039 & 3.767 & $1.068-13.284$ \\
Diabetes & $\mathrm{NE}$ & & & Diabetes & $\mathrm{NE}$ & & \\
MBP & $\mathrm{NE}$ & & & MBP & NE & & \\
WBC & $\mathrm{NE}$ & & & WBC & NE & & \\
BUN & .036 & 1.256 & $1.014-1.555$ & BUN & .023 & 1.298 & $1.036-1.625$ \\
P & .000 & 6965.965 & $576.828-84123.332$ & CaxP & .000 & 1.338 & $1.231-1.454$ \\
Ca or adjusted Ca & $\mathrm{NE}$ & & & & & & \\
\hline
\end{tabular}

NE: not entry. Method: Bachward LR. N=100 in WMHs. 
Table 6. Multivariate analysis for factors associated with CMBs by Binary Regression.

\begin{tabular}{|c|c|c|c|c|c|c|c|}
\hline & & Mod & $\mathrm{el2}$ & & & Mode & \\
\hline & $\mathrm{P}$-value & OR & $95 \% \mathrm{CI}$ & & P-value & OR & $95 \% \mathrm{CI}$ \\
\hline Age & .004 & 1.057 & $1.017-1.097$ & Age & .001 & 1.068 & $1.027-1.121$ \\
\hline Hypertension & $\mathrm{NE}$ & & & Hypertension & $\mathrm{NE}$ & & \\
\hline Diabetes & $\mathrm{NE}$ & & & Diabetes & $\mathrm{NE}$ & & \\
\hline MBP & $\mathrm{NE}$ & & & MBP & $\mathrm{NE}$ & & \\
\hline WBC & $\mathrm{NE}$ & & & WBC & $\mathrm{NE}$ & & \\
\hline BUN & $\mathrm{NE}$ & & & BUN & .047 & 1.248 & $1.003-1.552$ \\
\hline $\mathrm{P}$ & .000 & 558.429 & 49.395-6313.236 & $\mathrm{Ca} \times \mathrm{P}$ & .000 & 1.213 & $1.121-1.312$ \\
\hline $\mathrm{Ca}$ or adjusted $\mathrm{Ca}$ & $\mathrm{NE}$ & & & & & & \\
\hline
\end{tabular}

NE: not entry. Method: Bachward LR. N=55 in CMBs.

Table 7. H-L test of model 2 and model 3 in subtypes of CSVD.

\begin{tabular}{lllll}
\hline & Scattered lacunes & Multiple lacunes & WMHs & CMBs \\
\hline Model 2 & 0.858 & 0.404 & 0.693 & 0.541 \\
Model 3 & 0.571 & 0.404 & 0.295 & 0.560 \\
\hline
\end{tabular}

Models with $\mathrm{p}$ value $>0.1$ means good calibration power.

\section{Figures}

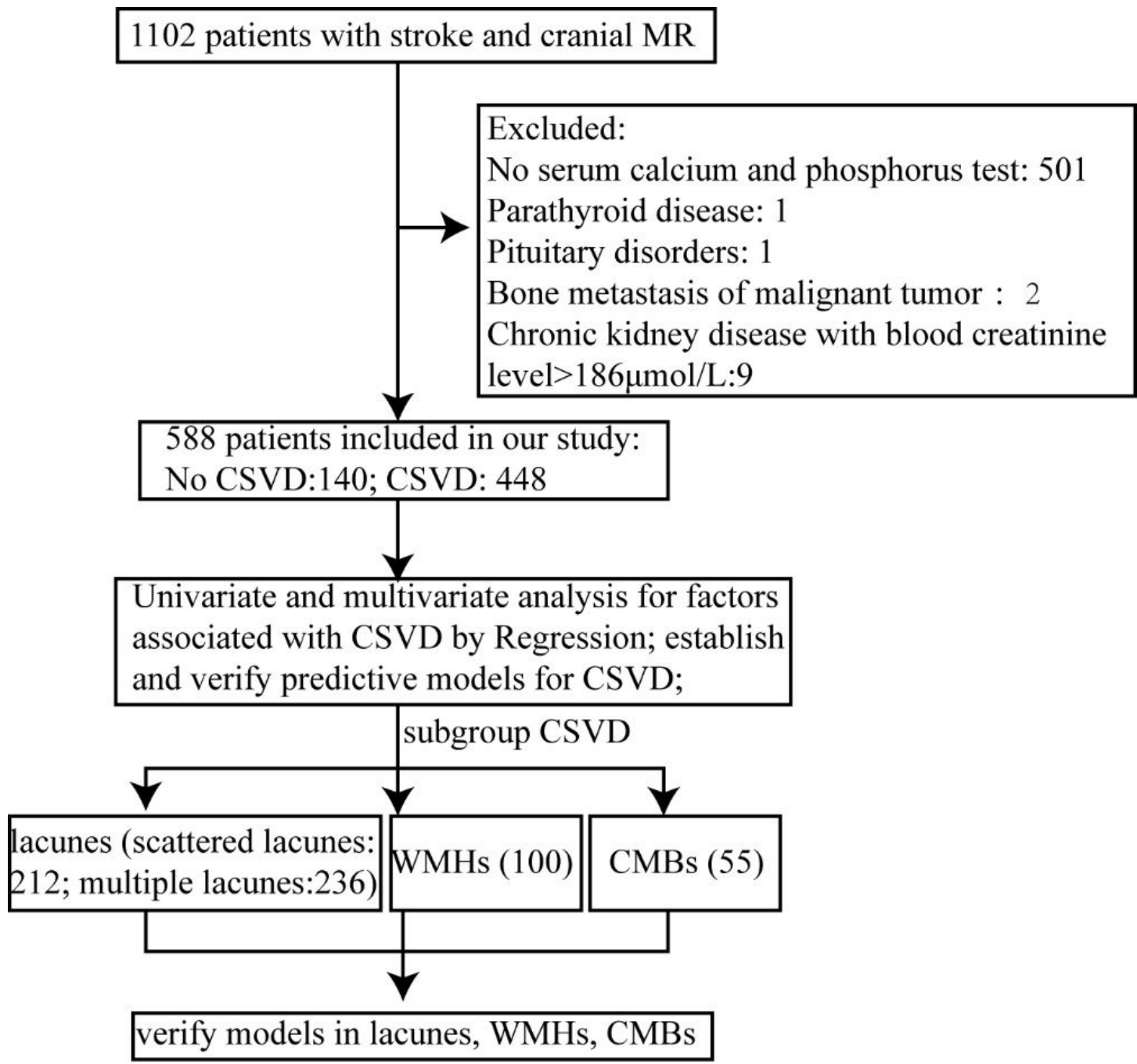


Flow diagram showing the patient selection and data analysis.

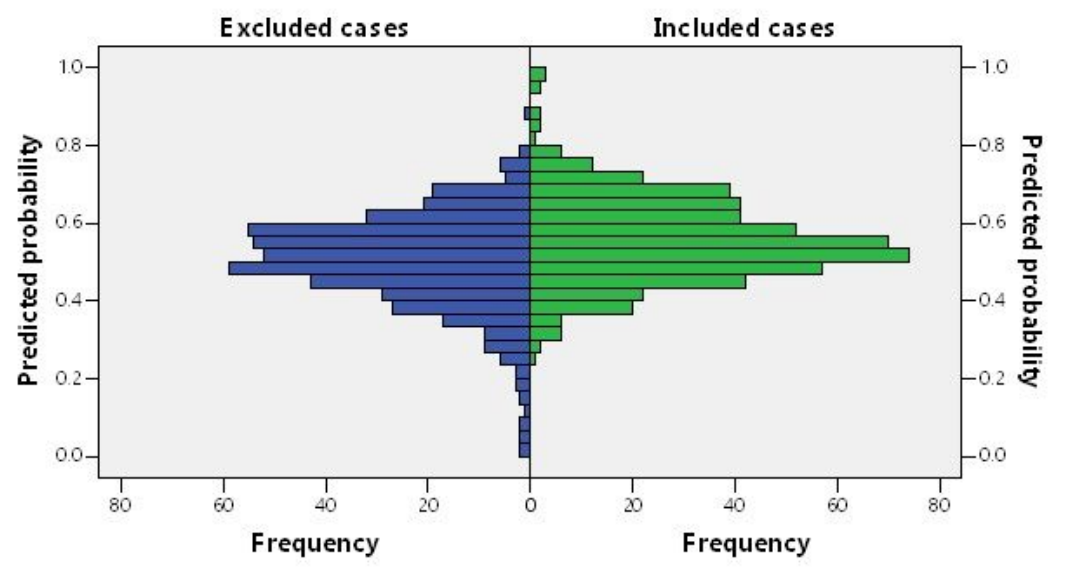

Figure 2

Propensity score of included cases and excluded cases, from demographic and clinical characteristics and routine serum test. Including age, gender, hypertension, diabetes, coronary artery disease, atrial fibrillation, hyperlipidemia, history of smoking and drinking alcohol, weight, NIHSS score at admission and discharge, MBP, LVEF, FBG, P2hPG, HbA1c\%, TC, LDL, HDL, cell count of WBC, neutrophil, lymphocyte, RBC, and PLT, HB, TP, albumin, PT, INR, APTT, FIB, BUN, Cr, HCY, TSH, FT3, FT4.

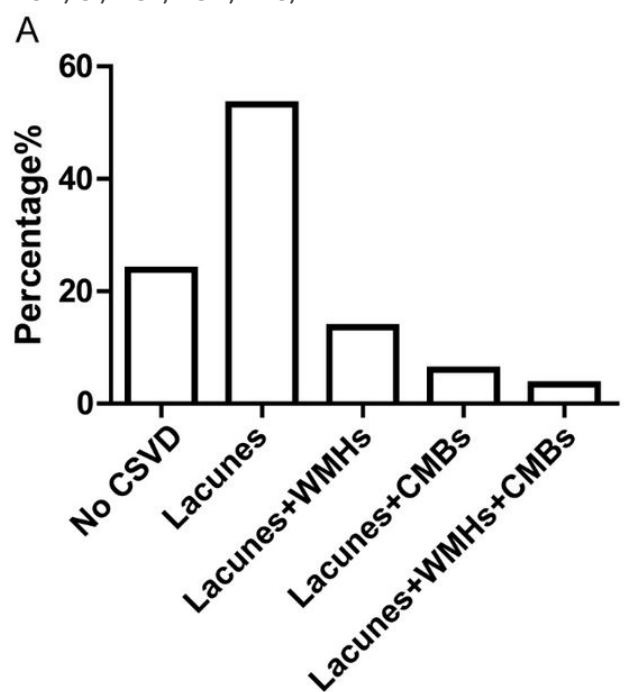

C

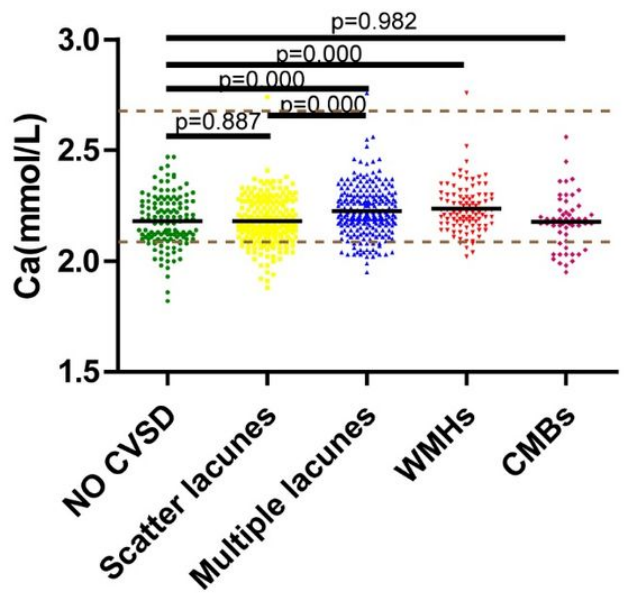

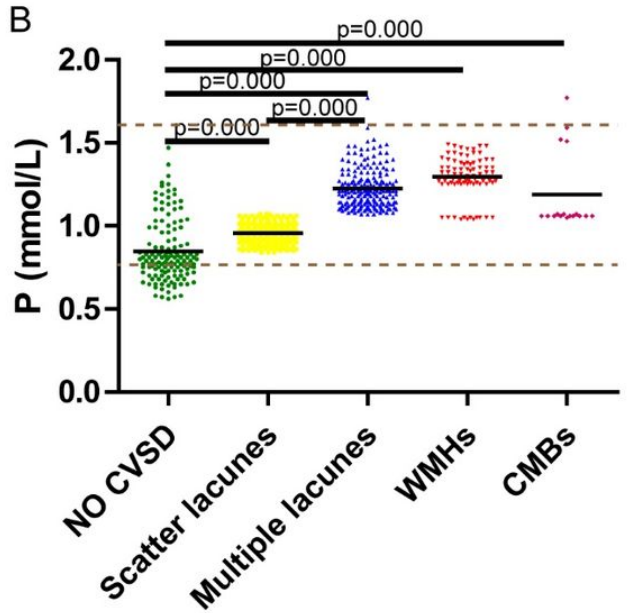

D

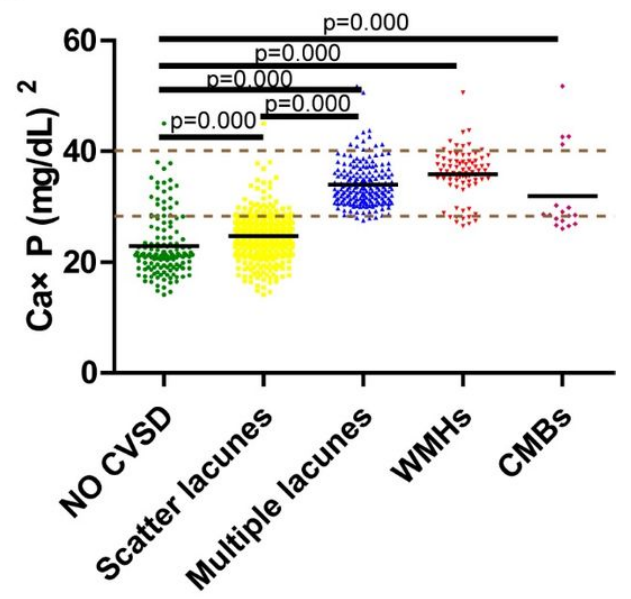

Figure 3

A Percentage of single or combined subtypes in CSVD. B-D Serum P, Ca, CaxP of subtypes in CSVD, including scattered lacunes, multiple lacunes, WMHs, and CMBs. Brown dotted lines is the upper and lower limits of normal values 


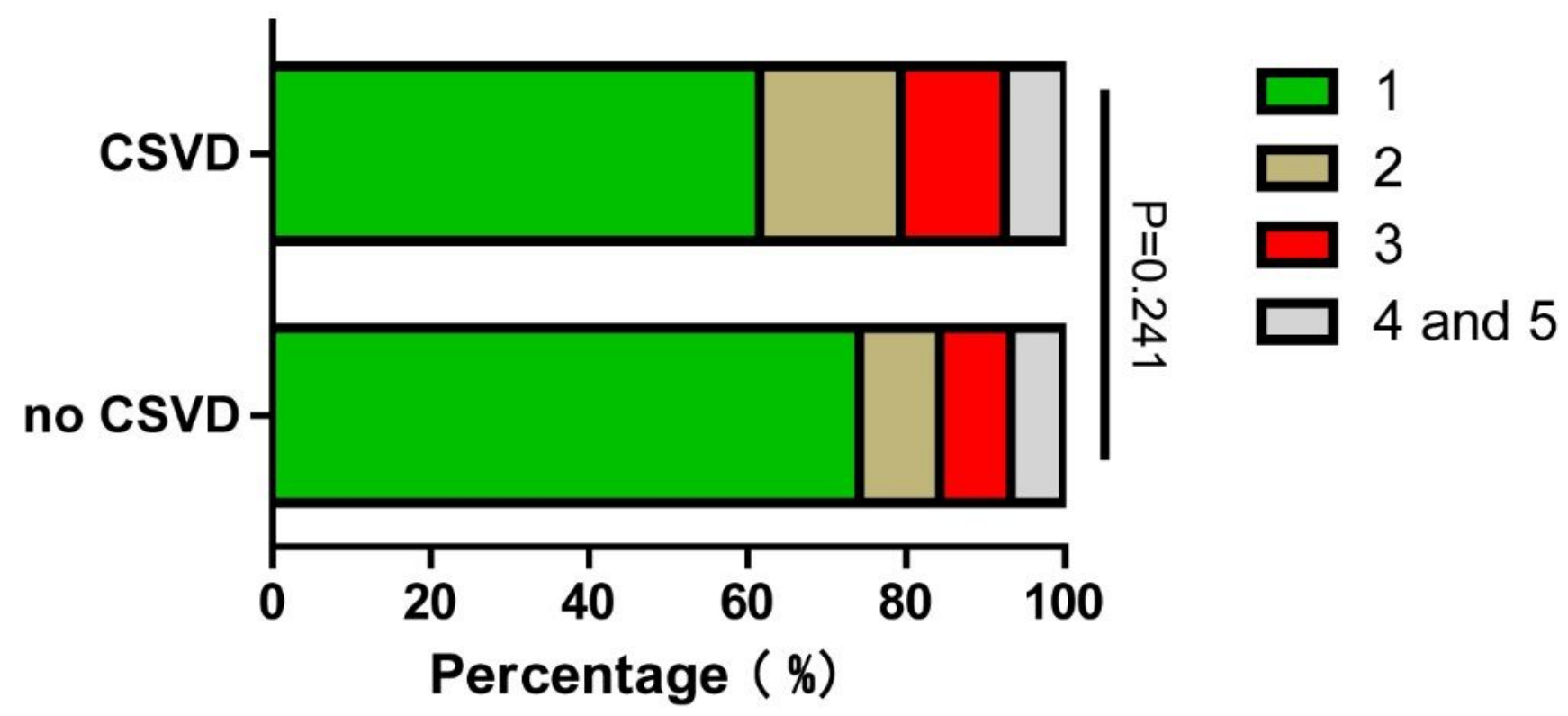

Figure 4

TOAST classification distribution of no CSVD and CSVD groups. TOAST classification: 1. Largeartery atherosclerosis. 2. Cardioembolism. 3. Small-artery occlusion. 4. Stroke of other determined cause. 5. Stroke of undermined cause.
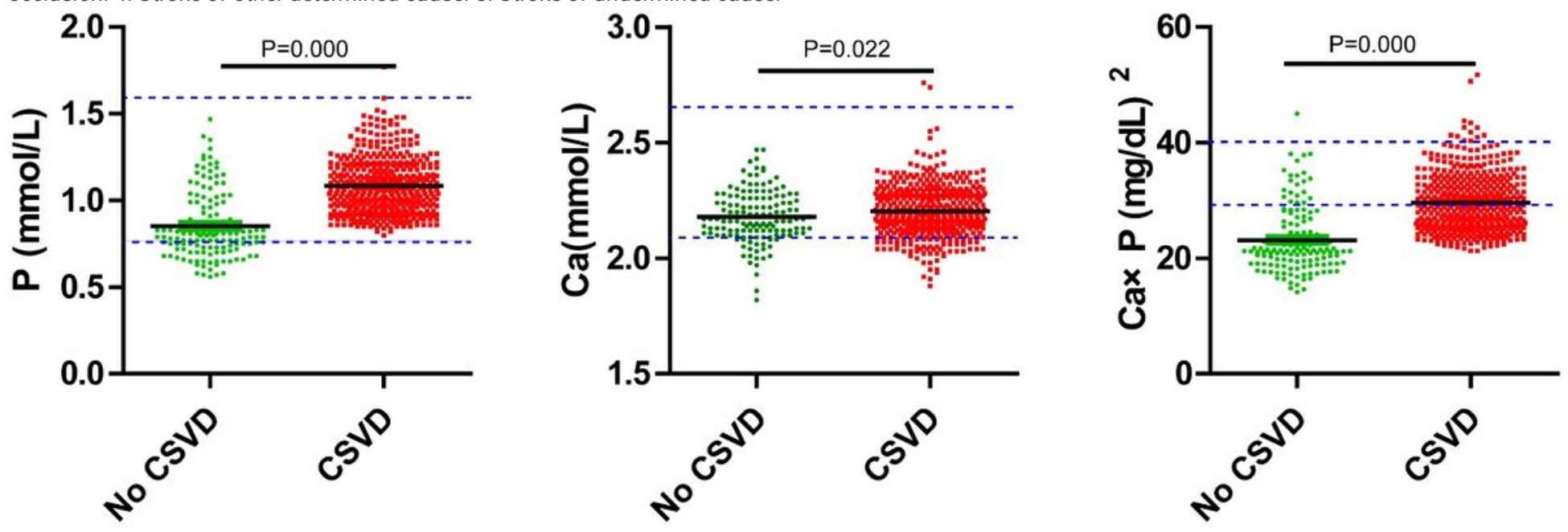

Figure 5

Serum $\mathrm{P}, \mathrm{Ca}, \mathrm{Ca} \times \mathrm{P}$, concentration of no CSVD and CSVD. Blue dotted lines is the upper and lower limits of normal values. 

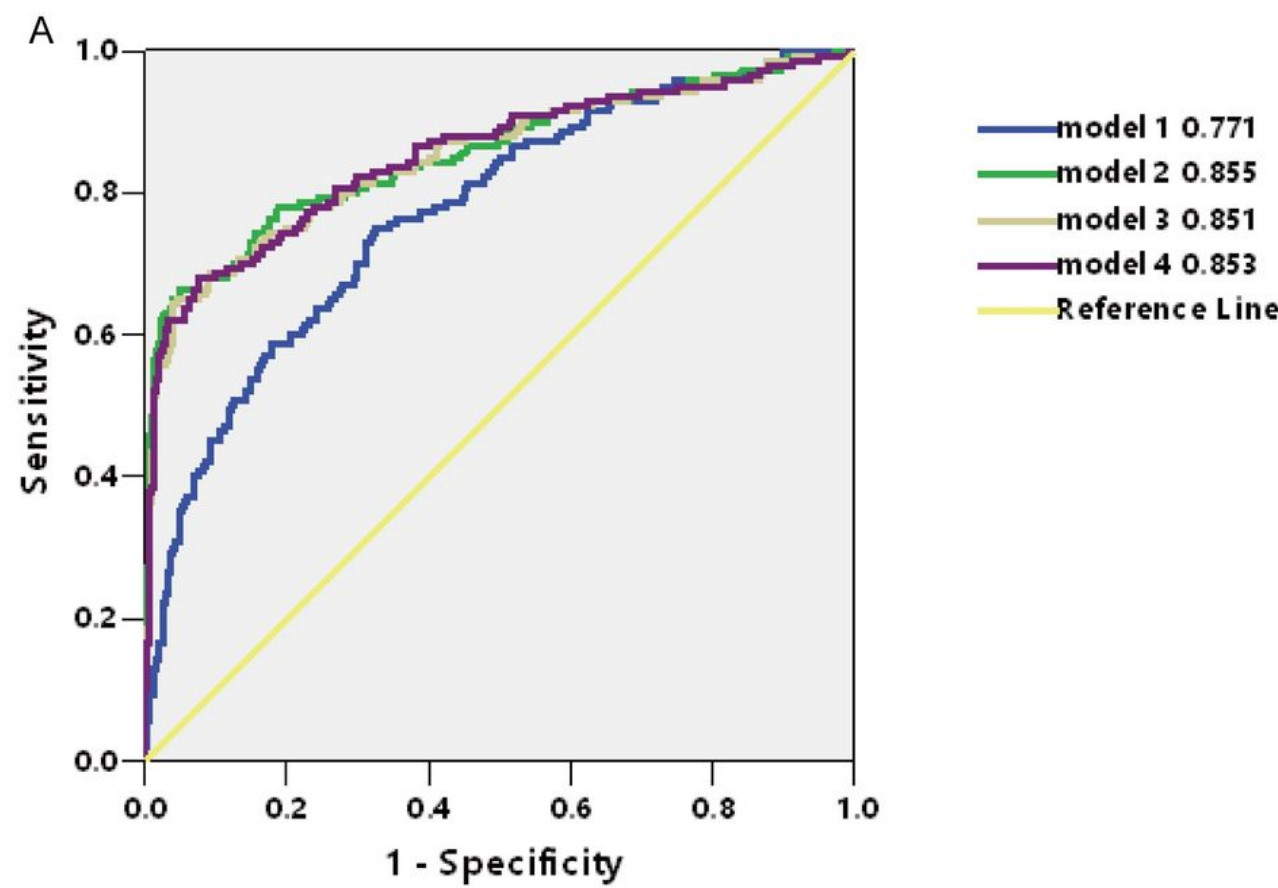

B

Model $1 \mathrm{P}=\mathbf{0 . 8 7 3}$
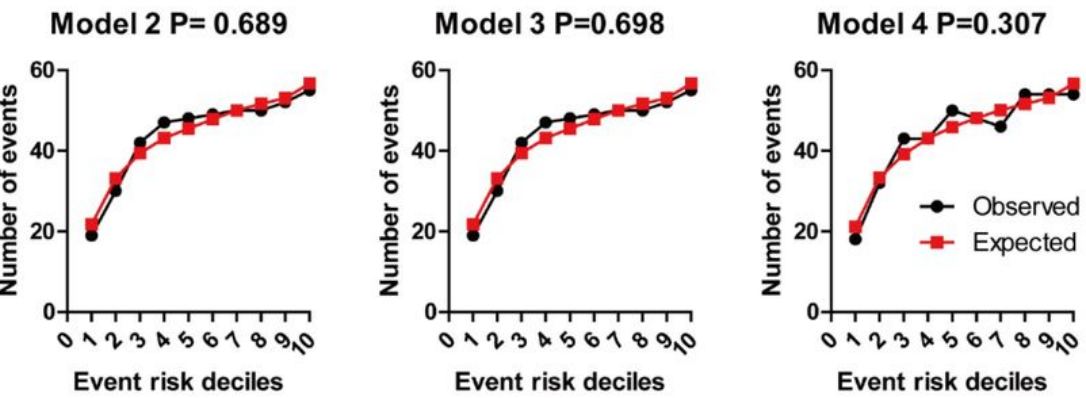

Figure 6

A AUCs of model 1-4. B H-L test of model 1-4. 
A

Points

$\begin{array}{llllllllll}0 & 10 & 20 & 30 & 40 & 50 & 60 & 70 & 80 & 90\end{array}$

age

hypertension

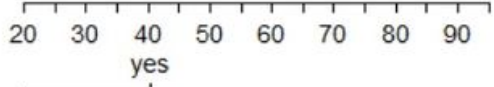

no

$\mathrm{P}$

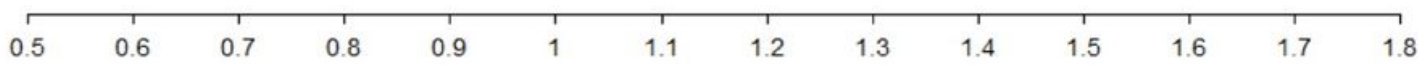

Total Points

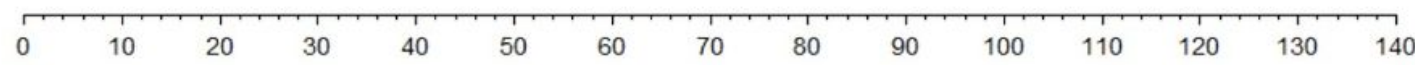

Probability of CSVD

$\begin{array}{llllllllll}0.05 & 0.1 & 0.2 & 0.30 .40 .50 .60 .7 & 0.8 & 0.9 & 0.95 & 0.99 & 0.999\end{array}$

B

Points

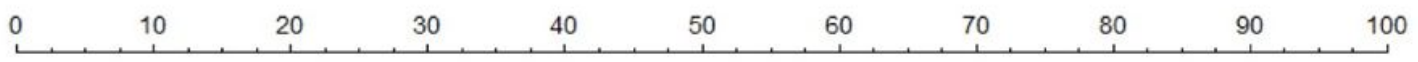

age

hypertension

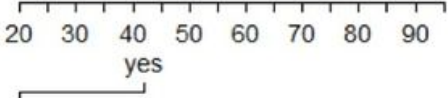

no

$\mathrm{CaxP}$

Total Points

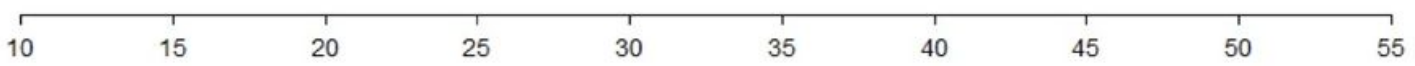

Probability of CSVD

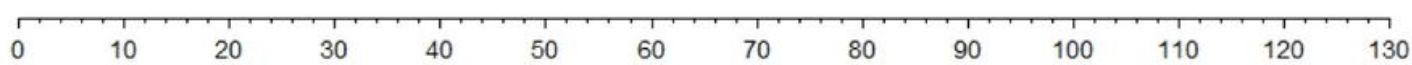

$\begin{array}{lllllllll}0.05 & 0.1 & 0.2 & 0.30 .40 .50 .60 .7 & 0.8 & 0.9 & 0.95 & 0.99 & 0.999\end{array}$

Figure 7

Nomograms of model 2 and model 3 for predicting the probability of CSVD. A model 2; B model 3
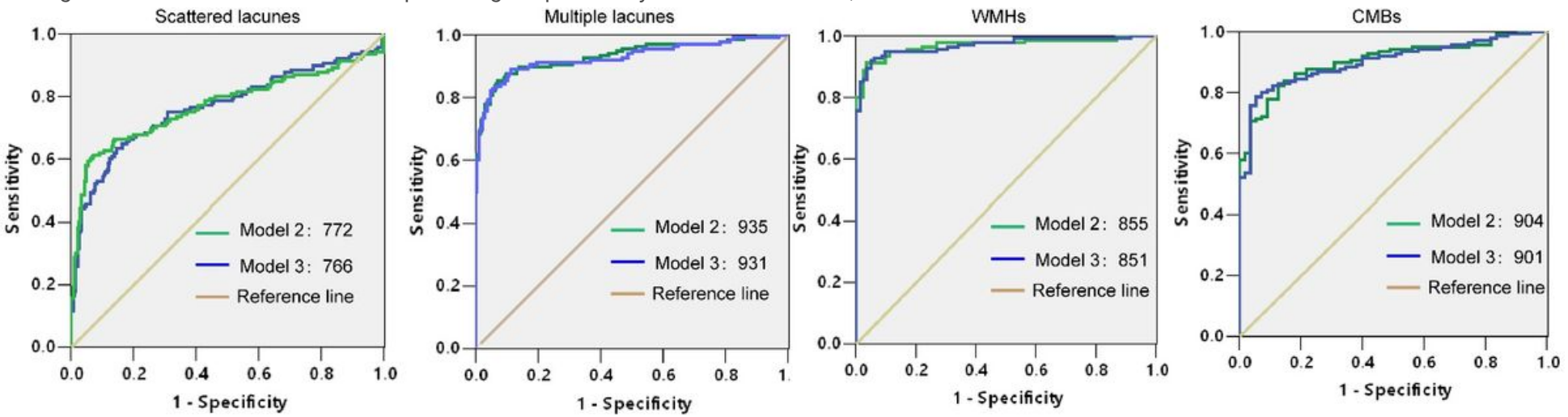

Figure 8

ROCs and AUCs of model2 and model3 in subtypes of CSVD. 